\title{
Degenerated Uterine Submucous Fibroid Prolapsing Through Lower Uterine Segment Caesarian Scar
}

\author{
Devika Gunasheela*, Jyothi Menon and Ashwin N \\ Gunasheela Fertility Centre, India
}

*Corresponding author: Devika Gunasheela, Gunasheela Fertility Centre, Bangalore, Karnataka 560 004, India

Submission: 皆February 19, 2018; Published: 漹 April 20, 2018

\section{Introduction}

Leiomyomas (Fibroids) are the most common benign tumors of the uterus and are found in approximately $2 \%$ of pregnant women. Degeneration of leiomyoma occurs secondary to loss of blood supply and is usually attributable to rapid growth associated with pregnancy. We report the management of a case of posterior wall large degenerated submucous fibroid prolapsing through the lower segment cesarean (LSCS) scar [1] (Figure 1).

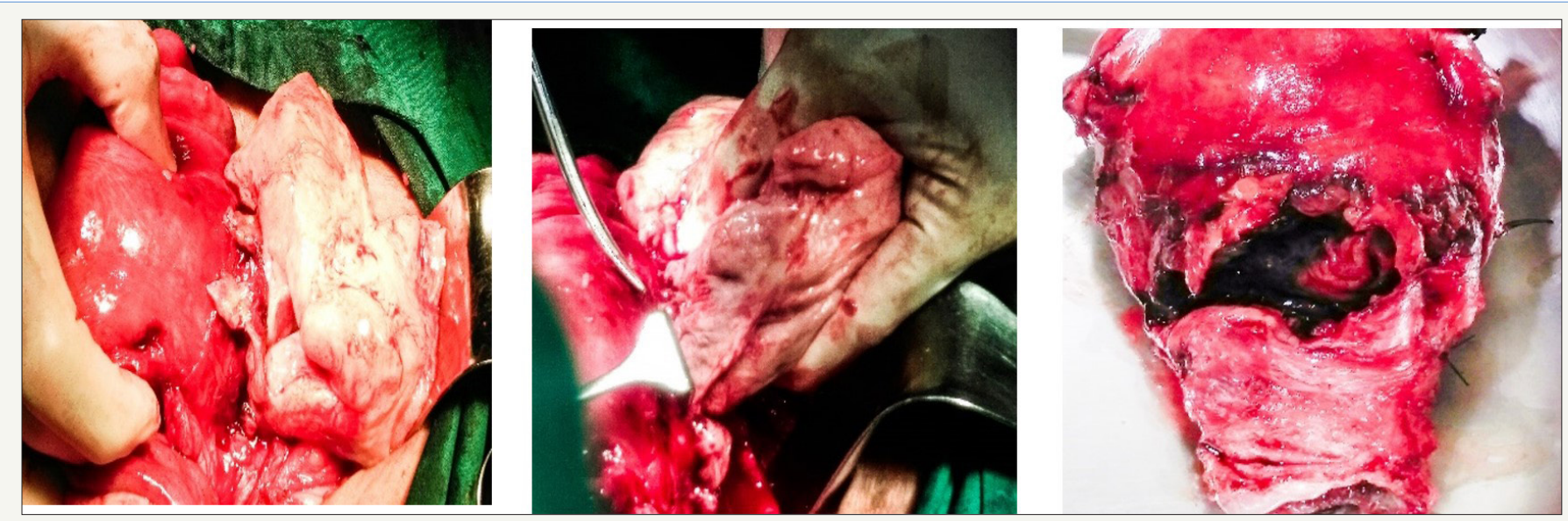

Figure 1

\section{Case Report}

A 32 year old woman para2 live2 presented with pain abdomen and menorrhagia on her $23^{\text {rd }}$ post-operative day after LSCS. At the time of admission patient was stable, her vitals were normal. Abdominal examination revealed a 20 weeks mass arising from pelvis, which is firm to cystic in consistency, tender with ill defined smooth edges. Per speculum and vaginal examination revealed $2 \mathrm{x}$ $3 \mathrm{~cm}$ prolapsed fibroid polyp. Cervical ring was felt all around the polyp with foul smelling, brown discharge [2].

Pre-operative blood investigations were within the normal limits. Ultrasound examination revealed $14 \times 10$ x 8 x cm submucous fibroid arising from posterior wall of uterus. MRI showed 14.3 x 8.1 x $10.2 \mathrm{~cm}$ submucous fibroid in uterine cavity. Patient was treated conservatively with intravenous analgesics and antibiotics, but bleeding and pain persisted. Hysteroscopy attempted and hysteroscope could not enter the uterus beyond the prolapsed uterine mass. Multiple vaginal washes were given. Patient and relatives were insisting on definitive surgery and hence decision for exploratory laparotomy and myomectomy was taken. The plan was to conserve the uterus and adnexa but intra-operative findings revealed a large degenerated submucous fibroid of size $14 \mathrm{x} 12 \mathrm{x}$ $10 \mathrm{~cm}$ prolapsing through the LSCS scar, causing ischemic injury to the whole of anterior wall of uterus. Bilateral ovaries and fallopian tubes were normal [3].

Total abdominal hysterectomy was planned and consent taken from husband and relatives, total abdominal hysterectomy was performed and bilateral ovaries conserved. Post operative period was uneventful and patient was discharged on $9^{\text {th }}$ post-operative day

Histopathological examination revealed leiomyoma with hyaline degeneration [4]. 


\section{Discussion}

Uterine myomas are observed in pregnancy more frequently now than in the past because many women are delaying child bearing till their late thirties, which is the time for greatest risk of myoma growth. Hyaline degeneration is suggested to begin with venus obstruction at the periphery of lesion, which induces hemorrhagic infraction and extensive necrosis that involves the entire lesion. Traditionally obstetricians are trained to avoid myomectomy during caesarean section due to severe haemorrhage and post-operative morbidity often necessitating hysterectomy. But some authors advocate it to be a safe and effective procedure [5].

There are various new techniques available to effectively control hemorrhage during cesarean myomectomy like uterotonic drugs, uterine tourniquet, bilateral uterine artery, uterine artery embolization, electro cautery, injecting diluted vasopressin in the fibroid. It is important that baby must be delivered prior to attempting myomectomy. Caesarean myomectomy can be performed in selected cases and it avoids the need for repeat laparotomy and its associated risks.

\section{Conclusion}

Caesarean myomectomy allows women to have a better obstetric outcome in future pregnancies, and to avoid hysterectomy.
It relieves symptoms associated with fibroid and negates the need for later surgery or sonographic follow-ups for the fibroid after delivery. It is a safe procedure in selected patients when performed in tertiary care hospitals by experienced obstetricians with newer measures available to curtail haemorrhage [5].

\section{References}

1. Novak ER, Woodruff JD (1979) Myomaand other benign tumours of the uterus. In: Novak ER, Woodruff JG (Eds.), Novak's Gynaecology and obstetric Pathology. WB Saunders, Philadelphia, USA, pp. 260-279.

2. Courbiere B, Carcopino X (2007) GynecologieObster Obstetrics. Vernazobres-Greco. Fibromesuterins, pp.359-365.

3. Okizuka H, Sugimura K, Takemori M, Obayashi C, Kitao M, et al (1993) MR detection of degenerating uterine leiomtomas. J Comput Assist Tomogr 17(5): 760-766.

4. Ueda H, Togashi K, Konshi I, Kataoka ML, Koyama T, et al. (1999) Unusual appearance of uterine lwimyomas: MR imaging findings and their histopathologic backgrounds. Radiographics 19 Spec No: S131-S145.

5. Kawakami S, Togashi K, Konishi I, Kimura I, Fukuoka M, et al. (1994) Red degeneration of uterine leimyoma: Mrappearance. J comput assist tomogr 18: 925-928.
Creative Commons Attribution 4.0 International License

For possible submissions Click Here

\section{Submit Article}

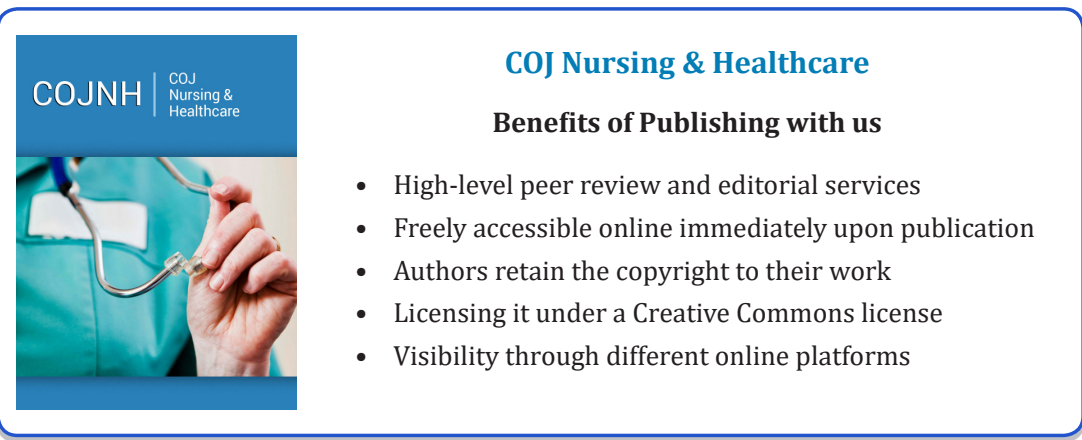

